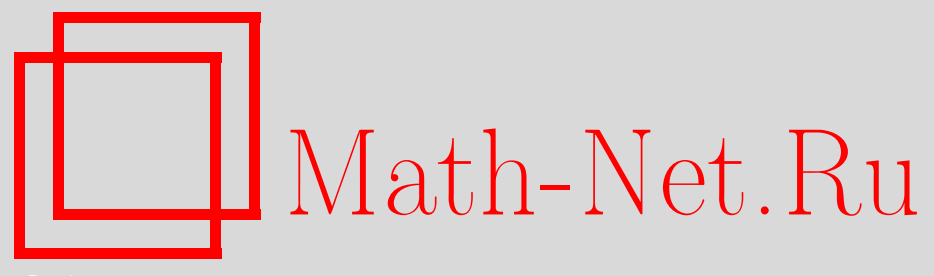

С. И. Дудов, А. Б. Коноплев, О приближении непрерывного многозначного отображения постоянными многозначными отображениями с шаровыми образами, $M a-$ тем. заметки, 2007, том 82, выпуск 4, 525-529

DOI: https://doi.org/10.4213/mzm3809

Использование Общероссийского математического портала Math-Net.Ru подразумевает, что вы прочитали и согласны с пользовательским соглашением http://www . mathnet.ru/rus/agreement

Параметры загрузки:

IP: 54.210 .77 .194

26 апреля 2023 г., 15:41:22

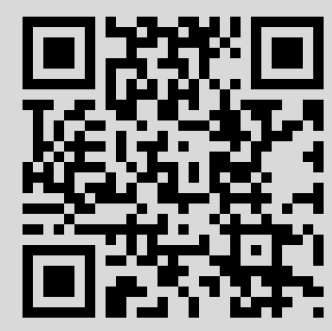


Том 82 выпуск 4 октябрь 2007

УДК 519.853

\section{О приближении непрерывного многозначного отображения постоянными многозначными отображениями с шаровыми образами}

\section{С. И. Дудов, А.Б. Коноплев}

Показано, что задача о наилучшем равномерном приближении в метрике Хаусдорфа непрерывного многозначного отображения с конечномерными выпуклыми компактными образами постоянными многозначными отображениями с шаровыми в смысле некоторой нормы образами может быть сведена к наглядной геометрической задаче. Эта задача требует построения шарового слоя наименьшей “толщины”, содержащего в себе множество, которое является дополнением выпуклого компакта до содержащего его другого выпуклого компакта.

Библиография: 8 названий.

1. В работе [1] непрерывное многозначное отображение (м.о.) с выпуклыми компактными образами равномерно в метрике Хаусдорфа приближалось постоянными м.о. с выпуклыми образами. В этой заметке мы также рассматриваем задачу, когда для приближения м.о. используются постоянные м.о., но с образами в виде шара некоторой нормы.

Пусть непрерывное на отрезке $[a, b]$ м.о. $F(\cdot)$ имеет выпуклые компактные образы $F(t)$ из действительного конечномерного пространства $\mathbb{R}^{p}$ :

$$
F(\cdot):[a, b] \Rightarrow K v\left(\mathbb{R}^{p}\right),
$$

$K v\left(\mathbb{R}^{p}\right)$ - пространство всех выпуклых компактов из $\mathbb{R}^{p}, n(x)$ - некоторая норма на $\mathbb{R}^{p}, B n(x, r)=\left\{y \in \mathbb{R}^{p}: n(x-y) \leqslant r\right\}$ - шар в этой норме с центром в точке $x$ и радиусом $r$,

$$
h(A, B)=\max \left\{\sup _{a \in A} \inf n(a-b), \sup _{b \in B} \inf _{a \in A} n(a-b)\right\}
$$

- расстояние Хаусдорфа между множествами $A$ и $B$ из $\mathbb{R}^{p}$.

Рассмотрим задачу

$$
\varphi(x, r) \equiv \max _{t \in[a, b]} h(F(t), B n(x, r)) \rightarrow \min _{x \in \mathbb{R}^{p}, r \geqslant 0}
$$

наилучшего равномерного на отрезке $[a, b]$ приближения м.о. $F(\cdot)$ постоянными м.о. с образами в виде шаров $B n(x, r)$. Основная цель - редуцировать задачу $(1)$ к одной наглядной геометрической задаче. 
Примем некоторые обозначения. Для выпуклого компакта $A \subset \mathbb{R}^{p}$ и множества $\Omega_{A}=\overline{\mathbb{R}^{p} \backslash A}$ определим функции

$$
\begin{gathered}
R(x, A)=\max _{y \in A} n(x-y), \quad \rho(x, A)=\min _{y \in A} n(x-y), \\
P(x, A)=\rho(x, A)-\rho\left(x, \Omega_{A}\right) .
\end{gathered}
$$

Свойства этих функций изучались, в частности, в [2]-[4]. Отметим, что они являются выпуклыми и конечными на $\mathbb{R}^{p}$. Поэтому, учитывая ограничения, накладываемые на м.о. $F(\cdot)$, также выпуклыми и конечными, а значит, и непрерывными [3] являются функции

$$
R^{F}(x)=\max _{t \in[a, b]} R(x, F(t)), \quad P^{F}(x)=\max _{t \in[a, b]} P(x, F(t)) .
$$

Под $\partial f(x)$ далее понимаем субдифференциал выпуклой функции $f(\cdot)$ в точке $x$, $A+B=\{a+b: a \in A, b \in B\}$ - алгебраическая сумма множеств $A$ и $B, o_{p}=$ $(0,0, \ldots, 0) \in \mathbb{R}^{p}$.

2. Существование решения задачи (1), как и в [1] для рассматриваемой там задачи, следует из непрерывности м.о. $F(\cdot)$ и компактности его образов. Приведем критерий ее решения.

ТЕОРема 1. Задача (1) эквивалентна задаче

$$
R^{F}(x)+P^{F}(x) \rightarrow \min _{x \in \mathbb{R}^{p}}
$$

При этом для того, чтобы пара $\left(x^{*}, r^{*}\right)$ являлась решением задачи (1), а точка $x^{*}$ решением задачи (2), необходимо и достаточно, чтобы выполнялись соотношения

$$
\begin{aligned}
& o_{p} \in \partial R^{F}\left(x^{*}\right)+\partial P^{F}\left(x^{*}\right), \\
& r^{*}=\frac{R^{F}\left(x^{*}\right)-P^{F}\left(x^{*}\right)}{2} .
\end{aligned}
$$

ДокАЗАТЕЛЬСтво. Как показано в [5], справедлива следующая формула расстояния Хаусдорфа между выпуклым компактом $A$ и шаром $B n(x, r)$ :

$$
h(A, B n(x, r))=\max \{R(x, A)-r, P(x, A)+r\} .
$$

Поэтому получаем представление целевой функции экстремальной задачи (1) в виде

$$
\varphi(x, r)=\max _{t \in[a, b]} \max \{R(x, F(t))-r, P(x, F(t))+r\}=\max \left\{R^{F}(x)-r, P^{F}(x)+r\right\} .
$$

Из определения функций $R^{F}(\cdot)$ и $P^{F}(\cdot)$ вытекает $R^{F}(x) \geqslant P^{F}(x)$ при всех $x \in \mathbb{R}^{p}$. Поэтому из (5) следует

$$
\min _{r \geqslant 0} \varphi(x, r)=\varphi(x, r(x))=\frac{R^{F}(x)+P^{F}(x)}{2}, \quad r(x)=\frac{R^{F}(x)-P^{F}(x)}{2} .
$$

Это говорит о том, что задача (1) эквивалентна задаче (2). При этом точка $x^{*}$ является решением задачи (2) тогда и только тогда, когда она является центром 
оптимального шара в задаче (1), а радиус этого шара выражается формулой (4). Как отмечалась выше, функции $R^{F}(x)$ и $P^{F}(x)$ являются выпуклыми на $\mathbb{R}^{p}$. Поэтому в соответствии с известным фактом из выпуклого анализа (см., например, [2; с. 142]) критерием того, что точка $x^{*}$ является решением задачи (2), является выполнение включения $o_{p} \in \partial\left(R^{F}\left(x^{*}\right)+P^{F}\left(x^{*}\right)\right)$. Теперь осталось заметить, что поскольку функции $R^{F}(\cdot)$ и $P^{F}(\cdot)$ являются выпуклыми и непрерывными, то, по теореме Моро-Рокафеллара [2; с. 78] справедливо равенство

$$
\partial\left(R^{F}(x)+P^{F}(x)\right)=\partial R^{F}(x)+\partial P^{F}(x) .
$$

Теорема доказана.

ЗАмЕЧАНИЕ 1. Используя формулы субдифференциалов функций $R(x, A)$ и $P(x, A)$ (см. [2; с. 163] и [5] соответственно) и правила субдифференциального исчисления выпуклых функций (конкретно [2; с. 87]), нетрудно выписать формулы субдифференциалов функций $R^{F}(x)$ и $P^{F}(x)$ для исследования задач $(1),(2)$ с помощью соотношения (3).

3. Редуцируем задачу (1) к более наглядной, с геометрической точки зрения, форме.

Обозначим через $F^{*}=\{F(t): t \in[a, b]\}$ полный образ м.о. $F(t)$,

$$
d=\max _{x, y \in F^{*}} n(x-y)
$$

- его диаметр, $A_{\lambda}=A+B n\left(0_{p}, \lambda\right)-\lambda$-окрестность множества $A$, со $A$ - выпуклую оболочку множества $A, F_{\lambda}(t)=(F(t))_{\lambda}$.

Нетрудно доказать следующие вспомогательные факты.

Лемма 1. Для любого выпуклого компакта $A$, положителъного $\lambda u x \in \mathbb{R}^{p}$ въполняется

$$
R\left(x, A_{\lambda}\right)=R(x, A)+\lambda, \quad P\left(x, A_{\lambda}\right)=P(x, A)-\lambda .
$$

Лемма 2. Для любых компактов $A, B u x \in \mathbb{R}^{p}$ выполняется

$$
\max \{R(x, A), R(x, B)\}=R(x, \operatorname{co}\{A, B\}) .
$$

Лемма 3. Если пересечение выпуклъх компактов $А$ и В не пусто, то для любой точки $x \in C$, где $C=A \cap B$, выполняется

$$
\max \{P(x, A), P(x, B)\}=-\rho\left(x, \Omega_{C}\right) .
$$

Теорема 2. Для любого $\lambda \geqslant 4 d$ задача (1) эквивалентна задаче

$$
R(x, A(\lambda))-\rho\left(x, \Omega_{B(\lambda)}\right) \rightarrow \min _{x \in B(\lambda)},
$$

где

$$
A(\lambda)=\operatorname{co}\left\{F_{\lambda}(t): t \in[a, b]\right\}, \quad B(\lambda)=\cap\left\{F_{\lambda}(t): t \in[a, b]\right\} .
$$

ДокАЗАтельство. Очевидно, при значениях $\lambda \geqslant d$ множество $B(\lambda) \neq \varnothing$. Тогда, с помощью лемм 1-3 и учетом условий, наложенных на м.о. $F(\cdot)$, нетрудно показать, что для всех $x \in B(\lambda)$ имеет место равенство

$$
R^{F}(x)+P^{F}(x)=R(x, A(\lambda))-\rho\left(x, \Omega_{B(\lambda)}\right) .
$$


Теперь остается показать, что при $\lambda \geqslant 4 d$ множество $B(\lambda)$ содержит все множество решений задачи (2). Возьмем произвольную точку $y_{0} \in F^{*}$ и рассмотрим множество

$$
G\left(y_{0}\right)=\left\{y \in \mathbb{R}^{p}: R^{F}(y)+P^{F}(y) \leqslant R^{F}\left(y_{0}\right)+P^{F}\left(y_{0}\right)\right\} .
$$

Из определений функций $R^{F}(\cdot)$ и $P^{F}(\cdot)$ легко следуют неравенства $R^{F}\left(y_{0}\right) \leqslant d$, $P^{F}\left(y_{0}\right) \leqslant d$, а также $P^{F}(y) \geqslant-d$ при любом $y \in \mathbb{R}^{p}$. Поэтому при любых $t \in[a, b]$ и $z \in F(t)$ имеем

$$
\begin{aligned}
G\left(y_{0}\right) & \subset\left\{y \in \mathbb{R}^{p}: R^{F}(y) \leqslant 3 d\right\}=\left\{y \in \mathbb{R}^{p}: \max _{z \in F(t)} n(y-z) \leqslant 3 d, t \in[a, b]\right\} \\
& \subset B n(z, 3 d)=z+\operatorname{Bn}\left(0_{p}, 3 d\right) \subset F^{*}+\operatorname{Bn}\left(0_{p}, 3 d\right) .
\end{aligned}
$$

С другой стороны, при любом $t \in[a, b]$ выполняется

$$
F_{d}(t) \equiv F(t)+B n\left(0_{p}, d\right) \supset F^{*}
$$

а следовательно, $F_{4 d}(t) \supset F^{*}+B n\left(0_{p}, 3 d\right)$, и тогда

$$
B(4 d) \equiv \bigcap\left\{F_{4 d}(t): t \in[a, b]\right\} \supset F^{*}+B n\left(0_{p}, 3 d\right) .
$$

Учитывая, что множество $G\left(y_{0}\right)$ содержит все множество решений задачи $(2)$, из $(8),(9)$ следует, что и множество $B(4 d)$ его также содержит. Теорема доказана.

ЗАмЕчаниЕ 2. Из наложенных на м.о. $F(\cdot)$ условий и из определений множеств $A(\lambda)$ и $B(\lambda)$ вытекает, что они являются выпуклыми компактами, причем $B(\lambda) \subset$ $A(\lambda)$. Значение функции $R(x, A(\lambda))$ выражает радиус наименьшего шара с центром в точке $x$, содержащего в себе множество $A(\lambda)$. А значение функции $\rho\left(x, \Omega_{B(\lambda)}\right)-$ радиус наибольшего шара с центром в точке $x \in B(\lambda)$, содержащегося в $B(\lambda)$. Поэтому с геометрической точки зрения задача (6) требует построения шарового слоя наименьшей "толщины” с центром из множества $B(\lambda)$, содержащего в себе множество $A(\lambda) \cap \Omega_{B(\lambda)}$.

ЗАмЕчаниЕ 3. Оценка сверху по включению для множества решений задачи (2) в виде множества $F^{*}+B n\left(0_{p}, 3 d\right)$, полученная в процессе доказательства, является грубой. С другой стороны, как показывают примеры, могут быть решения, которые не содержатся в со $F^{*}$.

4. В качестве примера рассмотрим м.о. вида

$$
F(t)=(1-t) A+t B, \quad t \in[0,1]
$$

где $A$ и $B$ - некоторые выпуклые компакты из $\mathbb{R}^{p}$.

Нетрудно показать, что в данном случае $F^{*}=\operatorname{co}\{A, B\}$ и при всех $x \in \mathbb{R}^{p}$ выполняется

$$
R^{F}(x)=\max \{R(x, A), R(x, B)\}, \quad P^{F}(x)=\max \{P(x, A), P(x, B)\} .
$$

Рассуждения, аналогичные приведенным при доказательстве теоремы 2, для м.о. вида (10) с учетом формул (11) приводят нас к справедливости следующего факта. 
Теорема 3. Задача (1) для м.о. вида (10) при всех значениях $\lambda \geqslant 4 d$ эквивалентна задаче

$$
R\left(x, \operatorname{co}\left\{A_{\lambda}, B_{\lambda}\right\}\right)-\rho\left(x, \Omega_{A_{\lambda} \cap B_{\lambda}}\right) \rightarrow \min _{x \in A_{\lambda} \cap B_{\lambda}} .
$$

Непосредственно из теоремы 3 получаем

СлеДСТвиЕ. Если $B \subset A$, то задача (1) для м.о. вида (10) при $\lambda \geqslant 4 d$ эквивалентна задаче

$$
R\left(x, A_{\lambda}\right)-\rho\left(x, \Omega_{B_{\lambda}}\right) \rightarrow \min _{x \in B_{\lambda}} .
$$

Из теоремы 1 и леммы 1 следует, что задача (1) эквивалентна соответствующей задаче о приближении м.о. $F_{\lambda}(\cdot)$ при $\lambda \geqslant 0$.

Теорема 2 фактически говорит о том, что при исследовании задачи (1) принципиальное значение имеет задача

$$
R(x, A)-\rho\left(x, \Omega_{B}\right) \rightarrow \min _{x \in \Omega_{B}}
$$

о построении минимального шарового слоя, содержащего множество, представляющего собой дополнение заданного выпуклого компакта $B$ до другого выпуклого компакта $A$, содержащего $B$. Для случая $A=B$ задача (12) становится задачей о построении шарового слоя наименьшей "толщины”, содержащего границу выпуклого компакта. Результаты по ее исследованию см. в [6] и в библиографии, указанной в [7], для случая, когда $n(x)=\|x\|$ - евклидова норма, а для произвольной нормы в [8]. Если $n(x)$ - евклидова норма, то, как показано в [7], эта задача эквивалентна задаче наилучшего приближения в метрике Хаусдорфа выпуклого компакта $A$ евклидовым шаром.

\section{СПИСОК ЦИТИРОВАННОЙ ЛИТЕРАТУРЫ}

[1] М. С. Никольский, "Об аппроксимации непрерывного многозначного отображения постоянными многозначными отображениями", Вестн. Моск. ун-та. Сер. 15. Вычисл. матем. и кибернетика, 1990, № 1, 76-80.

[2] Б.Н. Пшеничный, Выпуклый анализ и экстремальные задачи, Наука, М., 1980.

[3] В. Ф. Демьянов, Л. В. Васильев, Недифференцируемая оптимизация, Наука, М., 1981.

[4] С. И. Дудов, "Субдифференцируемость и супердифференцируемость функции расстояния", Матем. заметки, 61:4 (1997), 530-542.

[5] С. И. Дудов, И. В. Златорунская, "Равномерная оценка выпуклого компакта шаром произвольной нормы", Матем. сб., 191:10 (2000), 13-38.

[6] Т. Боннезен, В. Фенхель, Теория выпуклых тел, Фазис, М., 2002.

[7] М.С. Никольский, Д. Б. Силин, "О наилучшем приближении выпуклого компакта элементами аддиала", Оптимальное управление и дифференииальные уравнения, Тр. МИАН, № 211, 1995, 338-354.

[8] С. И. Дудов, "Об оценке границы выпуклого компакта шаровым слоем", Изв. Сарат. ун-та. Новая сер., 1:2 (2001), 64-75.

\section{С. И. Дудов}

им. Н. Г. Чернышевского

E-mail: dudovSI@info.sgu.ru

\section{А. Б. Коноплев}

Саратовский государственный университет

им. Н. Г. Чернышевского

E-mail: alexey.konoplev@gmail.com 